\title{
Influence of Rotation on Heat Transfer from a Two-Pass Channel with Periodically Placed Turbulence and Secondary Flow Promoters
}

\author{
S. DUTTA, J.-C. HAN, Y.-M. ZHANG \\ Department of Mechanical Engineering, Texas A\&M University, College Station, TX 77843-3123
}

\begin{abstract}
In a stationary duct, ribs placed at an angle oblique to the main flow direction are more effective in heat transfer enhancement than the ribs placed perpendicular to the flow. Obliquely placed ribs, besides tripping the boundary layer, produce secondary flow patterns to increase heat transfer from the surfaces. Ducts rotating about an axis perpendicular to their own also develop secondary flows. These two secondary flows, produced by oblique ribs and rotation, interact with each other and develop a new heat transfer pattern that is different from those produced by oblique ribs or by rotation alone. This paper uses two types of rib configurations $\left(60^{\circ}\right.$ parallel ribs and $60^{\circ}$ staggered ribs) as turbulence and secondary flow promoters. The local and surface averaged Nusslt numbers are presented for both stationary and rotating conditions. This experimental study is conducted on a two-pass square channel with two opposite rib roughened surfaces (leading and trailing sides). All the walls are maintained at the same temperature. The heat transfer results in a rotating condition show that the $60^{\circ}$ staggered ribs are more effective in the first pass but the $60^{\circ}$ parallel ribs do better in the second pass.
\end{abstract}

Key Words: Ribbed duct; Rotation; Turbine blade coolant passage

W ITH the increase of operating costs and sensitivity to environmental pollution, there is a growing necessity to increase the thermal efficiency of turbines. One way to do this is to increase the inlet gas temperature. Therefore, turbine blades that can withstand higher thermal loads are in greater demand. Standard metal blades with special cooling techniques are employed in turbines since no other material now available can simultaneously withstand such high levels of heat load and thermalmechanical stress. Turbine blades require sophisticated external and internal cooling techniques to sustain the stress-handling capabilities of the blades in a very hot operating environment.

Figure 1 shows the sectional view of a typical turbine blade. A two-pass rotating channel simulates the conditions of internal serpentine cooling passages of turbine blades. Earlier studies on cooling passages were based on stationary models, which were easier to construct and work on. It was observed that surface heat transfer in stationary ducts can be augmented by casting rib turbulators (see Han [1984]; Han et al. [1985, 1991]). Unlike a stationary duct flow situation, rotation introduces Coriolis and centrifugal buoyancy effects in the flow resulting in asymmetric velocity and turbulence intensity profiles (Johnston et al. [1972]). Therefore, to understand and improve internal cooling of turbine blades it is necessary to investigate the heat transfer patterns in an actual rotating frame.

There are fewer experimental studies on rotating channels than on stationary ducts with similar geometrical features. Han and Zhang [1992] covered most of the important related publications on rotating channels to that date. Therefore, a concise discussion on the previous works is given here. Initial studies were conducted on the effects of rotation on heat transfer characteristics in smooth channels with radial outward flow by Mori et. al. [1971], Clifford et al. [1984], Harasgama and Morris [1988], Guidez [1989], Morris and Ghavami-Nasr [1991], and Soong et al. [1991]. After the initial investigations, more complicated test cases were reported with roughened heat transfer surfaces and with multipass serpentine 


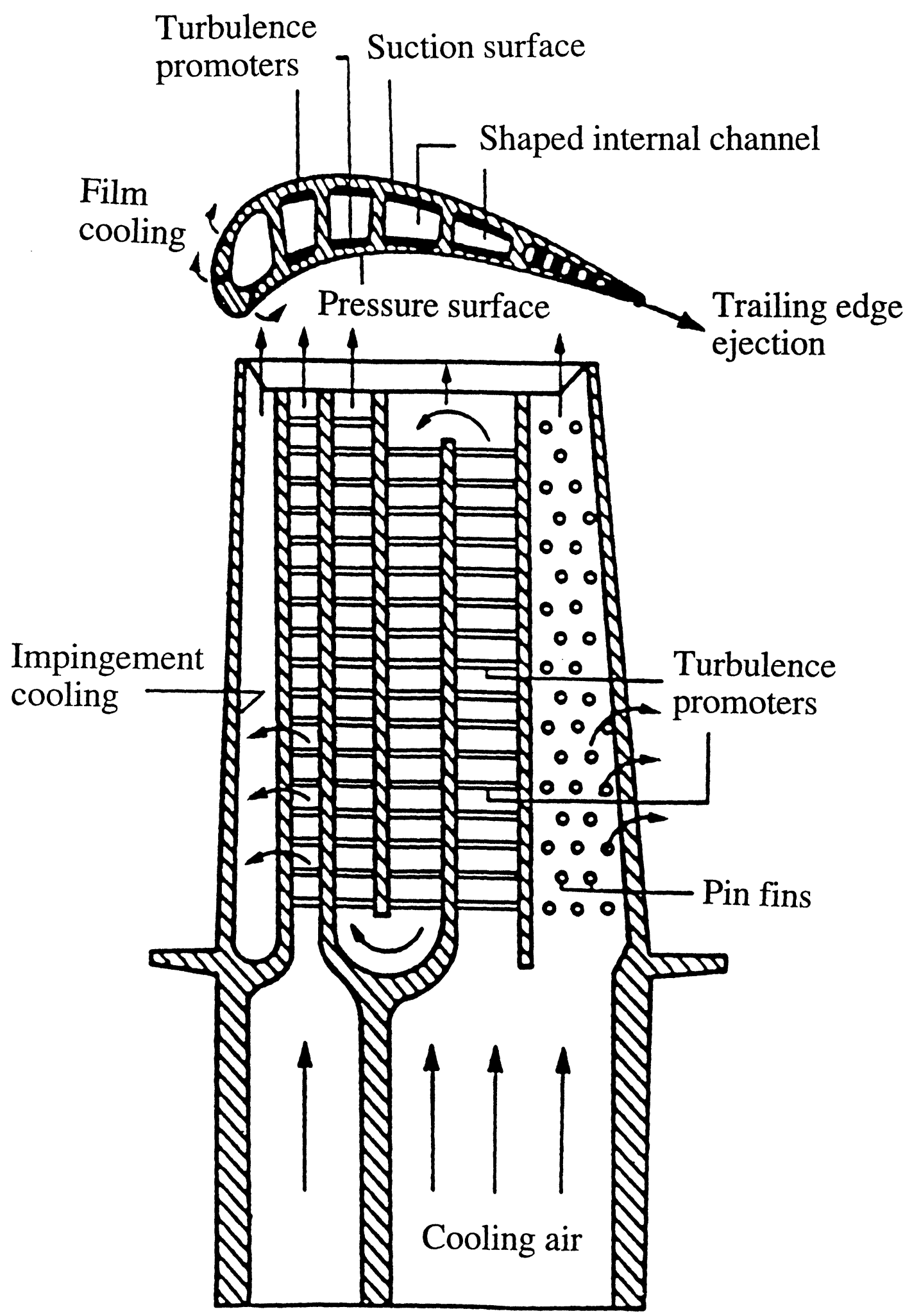

FIGURE 1 Schematic of the turbine blade coolant passages (from Parsons et al. [1994]). 
passages. Taslim et al. [1991a, 1991b] investigated heat transfer in a rotating duct with two opposite ribbed walls. In a series of publications Wagner et al. [1991a, 1991b, 1992] and Johnson et al. [1992] systematically explored the rotational effects on local heat transfer in serpentine coolant passage with and without ribs. Yang et al. [1992] and Zhang et al. [1993a] showed heat transfer distribution in a smooth and ribbed four-pass rotating serpentine flow passage.

Han and coworkers have been engaged in ribbed-duct heat transfer experiments for more than a decade. After successfully completing a series of rib configurations on a stationary duct, the emphasis is now shifted to a rotating channel with a two-pass flow passage. Han et al. [ , 1994] reported the effects of different surface heating conditions (uniform wall temperature and uniform wall heat flux) in rotating flow with smooth walls. The first-pass trailing and second-pass leading surfaces showed enhancement in Nusselt numbers with rotation. Unlike the first-pass trailing and the second-pass leading surfaces, the firstpass leading and the second-pass trailing surfaces showed a decrease in Nusselt numbers with an increase in the rotation number. The reduction in Nusselt numbers on the first-pass leading and the second-pass trailing surfaces make them the critical surfaces for possible burn out. Ribs were introduced in the leading and trailing sides of the two-pass channel to increase heat transfer. Parsons et al. [1994] and Zhang et al. [1993b] reported the results. It was seen that heat transfer increases with the addition of ribs, but the increase from the smooth wall condition differs in the leading and trailing sides of the channel. The rotation number was the key influence in developing the difference between leading and trailing side Nusselt numbers.

In one stationary test case, Han et al. [1991] studied the heat transfer augmentation in stationary square channel with parallel, crossed, and V-shaped ribs. It was shown that V-shaped ribs can increase the heat transfer rate threefold over the heat transfer rate from the smooth wall case. The heat transfer for V-shaped and $60^{\circ}$ parallel ribs were better than $90^{\circ}$ transverse ribs for a wide range of Reynolds numbers. This paper tests the effect of rotation on different rib configurations that, in a stationary frame, showed significant improvements over standard $90^{\circ}$ rib (Han et al. [1991]).

The present experimental work uses two types of rib configurations: $60^{\circ}$ parallel rib and $60^{\circ}$ staggered rib. These rib configurations are of special interest because ribs placed at an angle to the coolant flow induce secondary flow patterns that interact with the secondary flow generated by rotation. The performance of $60^{\circ}$ staggered ribs in a rotating channel are compared with the standard $90 \circ$ transverse and parallel ribs placed at a $60^{\circ}$ angle to the flow. Figure 2 shows the different rib configurations used and referred to in this experimental work. The smooth duct results of Han et al. [1993], the $90^{\circ}$ transverse rib of Parsons et al. [1994], and the $60^{\circ}$ parallel rib of Zhang et al. [1993b] are included to compare the performance of the $60^{\circ}$ parallel rib and the $60^{\circ}$ staggered rib of the present work. Note that the layout of the $60^{\circ}$ parallel rib of this work and the $60^{\circ}$ parallel rib of Zhang et al. [1993b] are different (Figures $2 \mathrm{c}$ and $2 \mathrm{~d}$ ). The ribs at the leading and trailing walls are aligned to be on top of each other.

Figure 3 shows the schematic of the vortex structures due to skewed rib configuration (no rotation) and rotation (smooth wall). From the rib orientations it is postulated that the $60^{\circ}$ parallel rib would induce a two-vortex structure and the $60^{\circ}$ staggered rib would generate a fourvortex structure. The staggered orientation would cause the vortex generated by one pair of leading-trailing $60^{\circ}$ staggered ribs to dominate the upstream pair's vortices. For clarity, Figure 3 a shows the stronger vortices in solid lines and the weaker vortices in broken lines. The vortex structure for rotation in a smooth channel is taken from Dutta et al. [1994]. In their numerical work, Dutta et al. [1994] observed two types of vortices for radially outward flow (Figure $3 b$ ). The second-pass (radially inward flow) vortices for rotation are extrapolated from the first-pass (radially outward flow) simulations. Figure 3 shows that the vortex structures of ribs and rotation are not aligned and would interact to give new complicated vortex structures. The new structure would be dependent on the relative strengths of the interacting vortices and would differ with Reynolds number and rotation number.

Local heat transfer results from the leading and trailing sides of the channel are presented in this paper. The surface averaged Nusselt number ratios are also included. The duct is rotated at an angular speed of $800 \mathrm{rpm}$ and the Reynolds number of the flow altered to get four different rotation numbers varying from 0.0352 to 0.352 . The four walls of the square channel are maintained at uniform temperatures at all axial locations.

\section{EXPERIMENTAL SET-UP}

Han et al. [1993] provide a detailed illustrated description of the experimental set-up. Therefore, only a brief description of the experimental facility is given here. Figure 4 shows the schematic of the test rig. Regulated compressed air is used as the working fluid. An orifice meter measures the mean flow rate passing through the test section, which rotates in a horizontal plane. There are two sets of slip ring units. One maintains a connection be- 


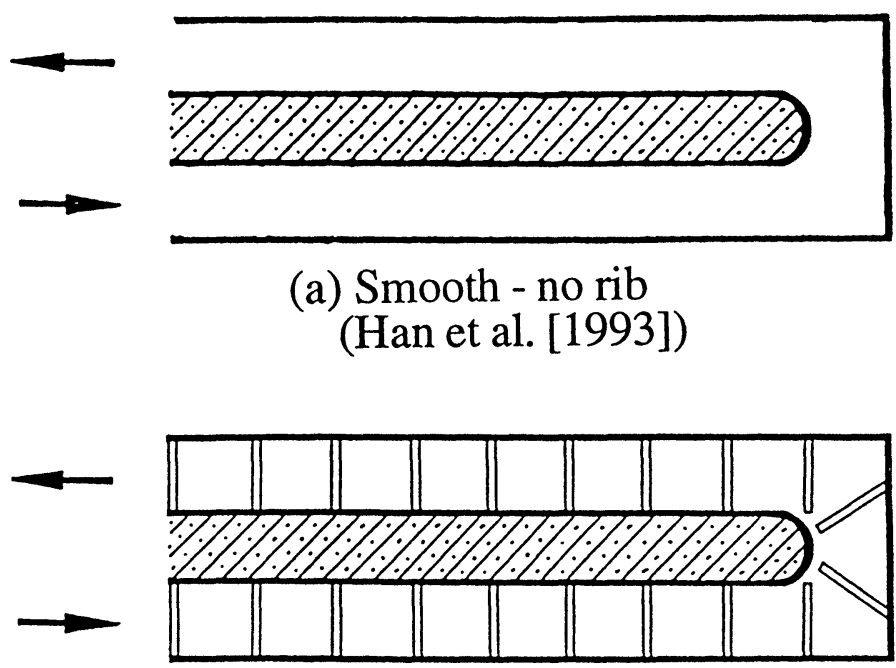

(b) $90^{\circ}$ Transverse rib (Parsons et al. [1994])

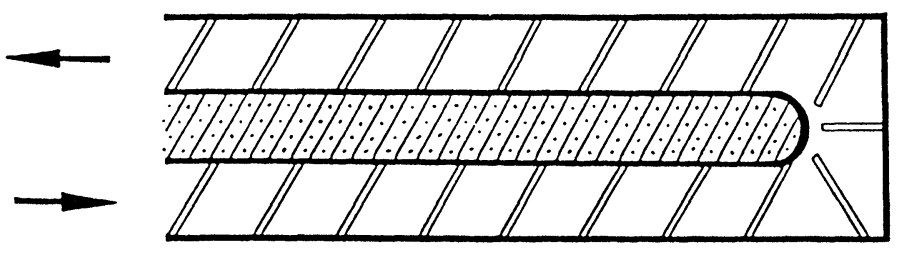

(c) $60^{\circ}$ Parallel rib

(Zhang et al. [1993b])

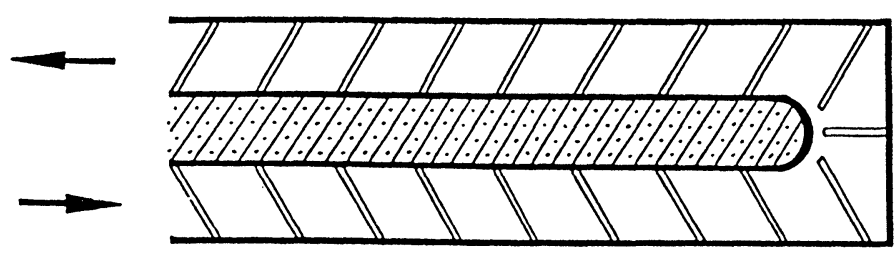

(d) $60^{\circ}$ Parallel rib

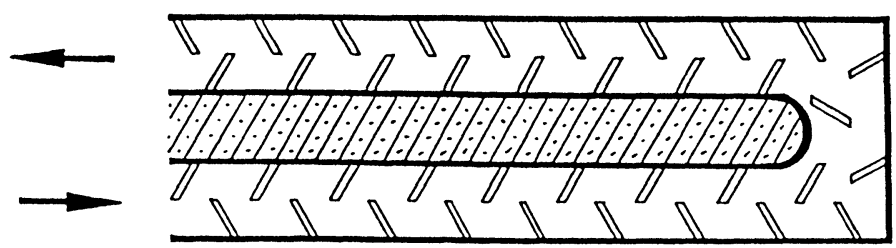

(e) $60^{\circ}$ Staggered rib

FIGURE 2 Rib configurations used and compared with. 

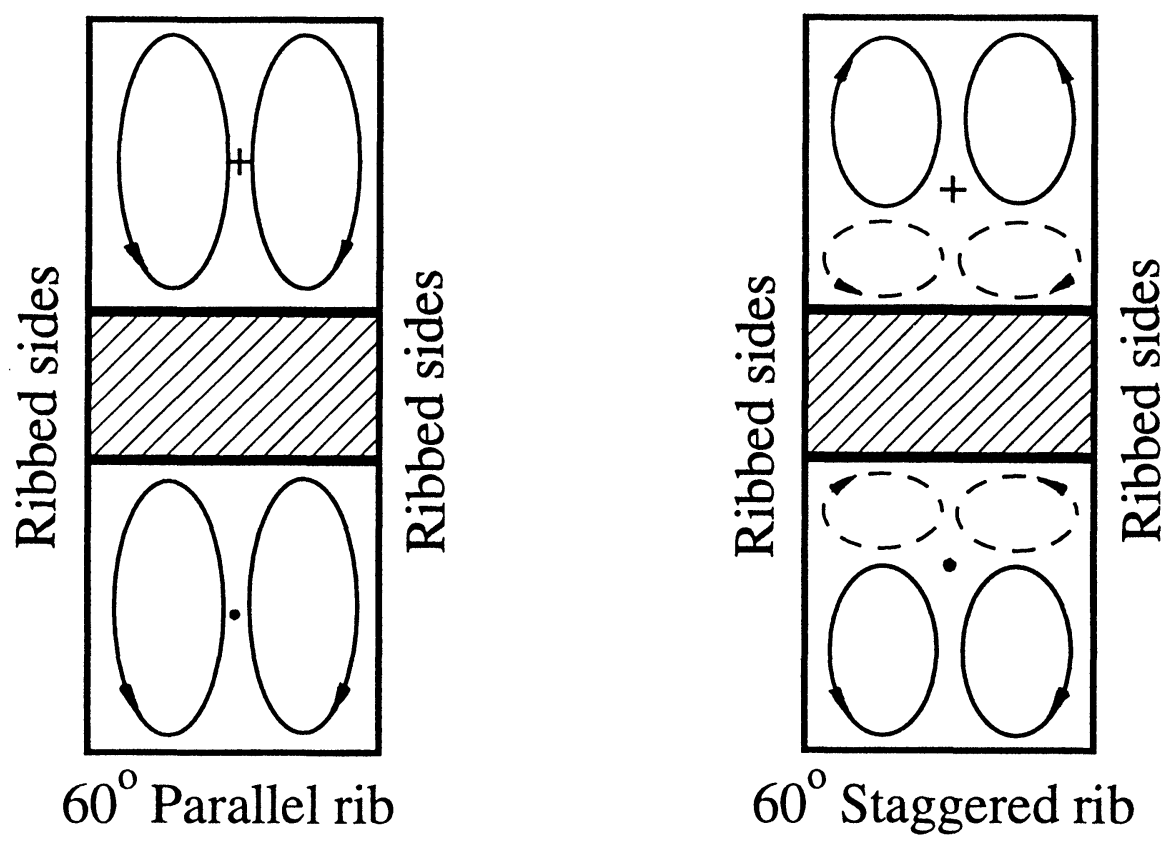

(a) Vortex structures due to the rib orientations at no rotation.

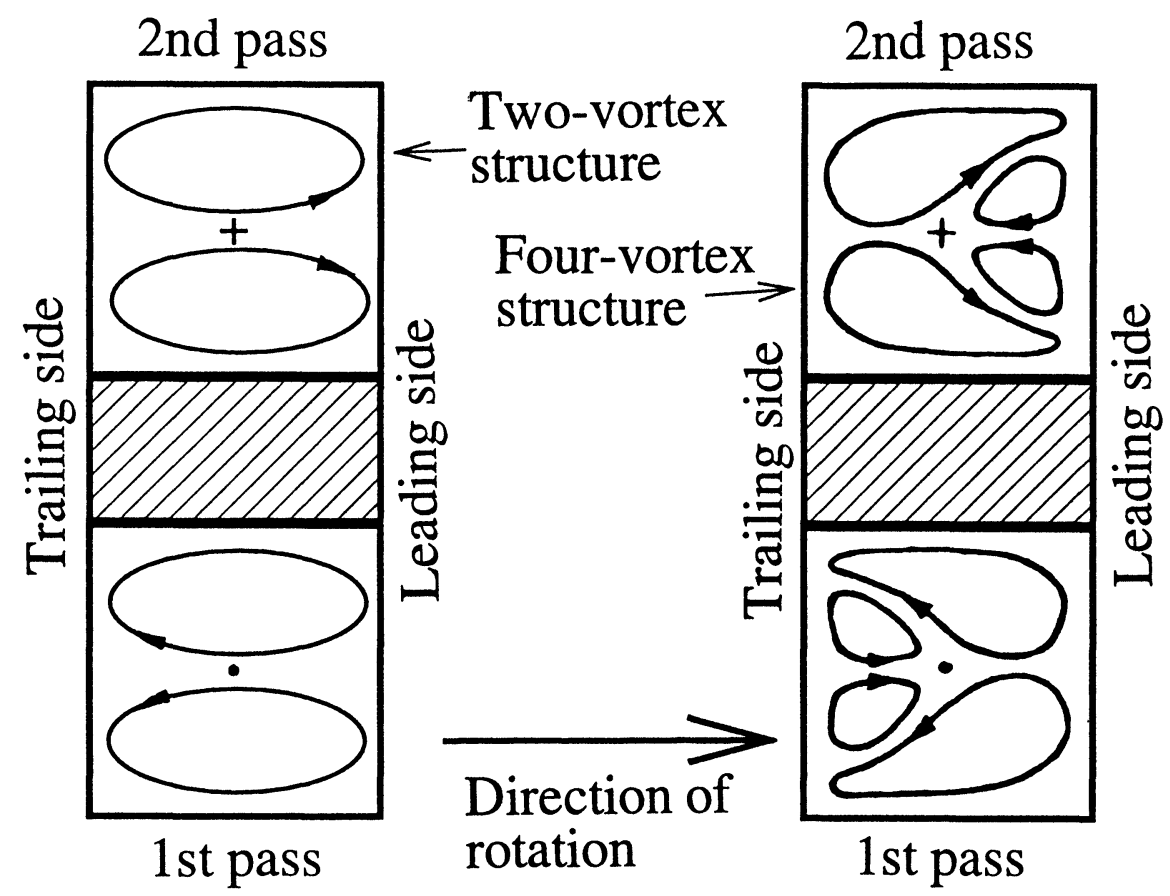

(b) Vortex structures due to rotation in a smooth duct.

FIGURE 3 Schematic of the secondary flow vortex structures generated by rib orientations and by rotation. 
tween thermocouples and the data logger interfaced to a personal computer. The other connects the power transformers to the heater units built in to the test section. A digital photo tachometer measures the rotation speed.

Each pass of the two-pass duct is $12.7 \mathrm{~mm}$ by $12.7 \mathrm{~mm}$ in cross-section. The flow in the first pass is radially outward, while the flow in the second pass is radially inward. Figure 5 shows a cut-away view of the two-pass test section with $60^{\circ}$ staggered ribs. To get local heat transfer coefficients, the duct is divided into twelve sections (Figure 5) with six in the fist pass and six in the second pass. The heater sections are two-hydraulic-diameters-long copper plates insulated from each other. Each of the nine walls (eight walls of the two passes and the turn) is insulated from each other and have independent heater coils powered by VARIAC transformers. The power input and thus the temperature of any surface can be adjusted to a desired level. The power to the heaters is adjusted so that the wall temperatures remain close to $40^{\circ} \mathrm{C}$ above ambient. Typical wall and bulk temperature profiles were given in Han et al. [1993].

The channel length to hydraulic diameter ratio is 24 , while each pass is 12 hydraulic diameters long connected by a sharp $180^{\circ}$ turn. The mean rotating arm length is 30 hydraulic diameters. The ribs are placed on the leading and trailing sides of the heated duct. Brass ribs of square crosssection are glued to the heater surfaces. The glue thickness is kept to less then $0.3 \mathrm{~mm}$. The rib height to hydraulic diameter ratio is 0.125 and the pitch to height ratio is 10. The angle between the coolant flow direction and the rib axis is $60^{\circ}$. The ribs on both the leading and trailing surfaces are exactly on top of each other and not staggered. Figures $2 \mathrm{~d}$ and $2 \mathrm{e}$ show the two rib configurations used in this paper. The arrows show bulk flow directions.

The experiment uses four Reynolds numbers: 2,500, $5,000,10,000$, and 25,000 . Variation of the mean flow velocity through the test section changes the Reynolds number. The duct, rotates at $800 \mathrm{rpm}$, resulting in rotation numbers of $0.352,0.176,0.088$, and 0.0352 .

\section{DATA REDUCTION}

The local heat transfer coefficient is calculated from the local net heat flux, the local wall temperature, and the local bulk mean air temperature difference as

$$
h=q_{\text {net }} /\left(T_{w}-T_{b}\right)
$$

Local net heat flux $\left(q_{\text {net }}\right)$ is calculated by subtracting heat loss from the power supplied to the heaters. Heat loss is estimated by running a separate test on the same test configuration but without air flow in the duct. Characteristic heat loss lines are obtained for each of the nine heater surfaces. For simplicity, all the heat flux calculations employ a smooth heater area with no rib surfaces included in the calculations. The local wall temperature $\left(T_{w}\right)$ is obtained from the thermocouple reading of that heater section. The local bulk temperature $\left(T_{b}\right)$ uses a linear interpolation between measured inlet and outlet bulk temperatures.

To reduce the influence of the flow Reynolds number on the heat transfer coefficient, the local Nusselt number is normalized by the Nusselt number for fully developed turbulent flow in smooth stationary circular pipe correlated by Dittus-Boelter/McAdams (Rohsenow and Choi [1961]) as

$$
N u / N u_{0}=(h D / k) /\left(0.023 \operatorname{Re}^{0.8} \operatorname{Pr}^{0.4}\right)
$$

Prandtl number (Pr) for air is 0.72 . Air properties are based on the average of the inlet and outlet bulk mean air temperatures.

The uncertainty of the local heat transfer coefficient depends on the uncertainties in the local wall and bulk air temperature difference, and net heat input for each heater section. This uncertainty increases for decreasing both the local wall to air temperature difference and the net heat flux. Based on the method described by Kline and McClintock [1953], The typical uncertainty in the Nusselt number is estimated to be less than 8 percent for Reynolds numbers larger than 10,000 . However the maximum uncertainty could be 25 percent for the lower heat transfer coefficient at the lowest Reynolds number tested $(\operatorname{Re}=2,500)$.

\section{RESULTS AND DISCUSSION}

Figures 6 and 7 show the local distribution of Nusselt number ratios at leading and trailing surfaces for rotating and nonrotating situations. Each data point presented is the averaged result over a heater section of two hydraulic diameters $(D)$ long. In a stationary duct both ribbed sides should have identical Nusselt number ratio profiles. Figures 6 and 7 show that there are differences in the local Nusselt number ratios between two ribbed sides especially in the first pass for both rib configurations. However, note that the profiles of the Nusselt number ratios are similar for the leading and trailing sides at nonrotating condition.

Figure 6 presents results for $\operatorname{Re}=5,000$. The stationary duct results in Figure 6 show the peak Nusselt numbers, for both rib configurations, occur at a downstream location of the inlet rather than at the entrance region of the heated duct. Earlier study of $60^{\circ}$ parallel rib in a stationary frame 


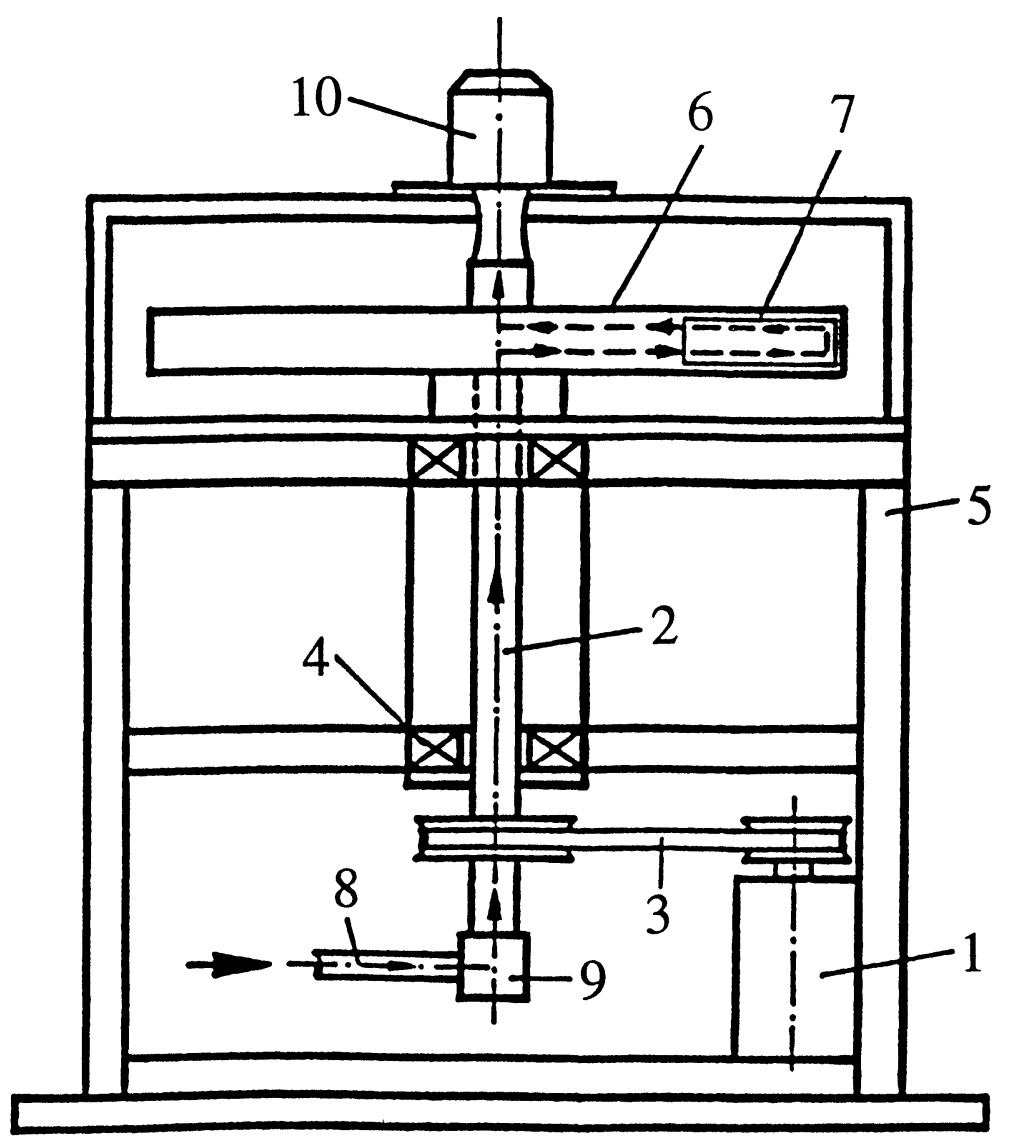

\section{Frequency controlled electric motor \\ 2. Rotating hollow shaft \\ 3. Belt drive \\ 4. Bearing \\ 5. Chasis of the experimental rig}

6. Horizontal rotating arm

7. Test section inside the rotating arm

8. Air supply

9. Rotary connector for air supply

10 . Slip ring assembly

FIGURE 4 Schematic of the rotating rig (from Han et al. [1993]).

by Han et al. [1991] also showed an increase in Nusselt number downstream of the entrance. However, the experimental set-up of Han et al. [1991] had a larger hydraulic diameter ( 2 inches) and the rib height to hydraulic diameter ratio was smaller than the ratio used in this work. In the first pass, the peak Nusselt numbers for both rib configurations, occur near $x / D=5$ and is believed to be due to the generation of secondary flows induced by the rib orientations. As the flow develops through the duct, the Nusselt number ratio decreases followed by an increase near the exit of the first pass. Nusselt number ratios increase at the exit of the first pass, $x / D=11$, because of the $180^{\circ}$ bend. The inlet of the second pass starts from the sharp bend and the Nusselt number profiles differ from those of the first pass. The stationary duct staggered rib does better than the other rib configuration in the second pass. Nusselt number ratios initially drop after the bend and then increase followed by a decrease (may be toward an equilibrium value). The initial drop is due to the decay of turbulence produced by the sharp $180^{\circ}$ bend. The rib 


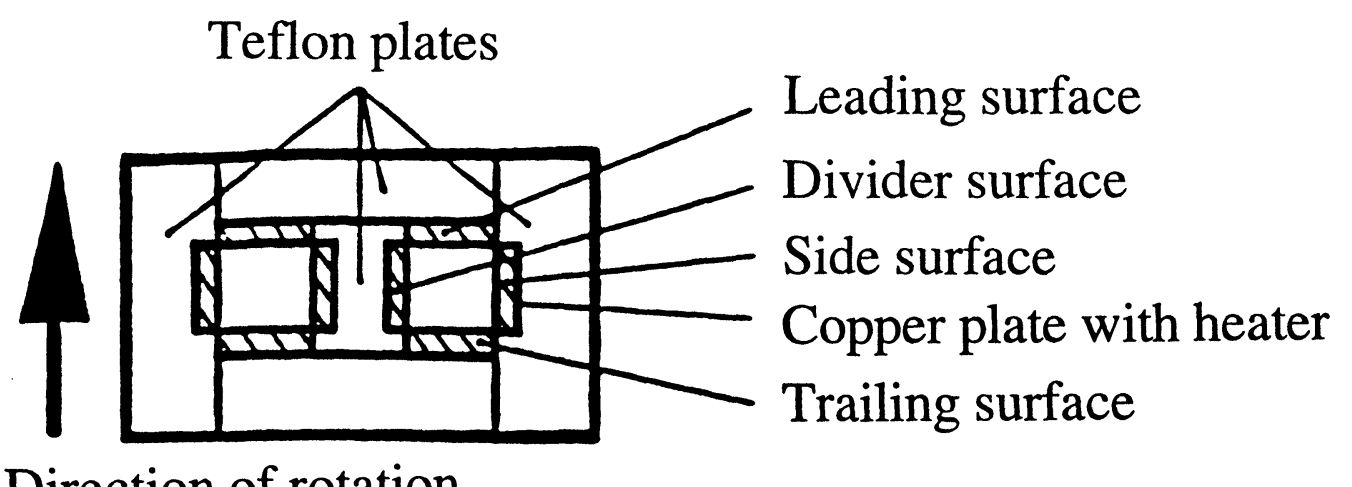

Direction of rotation
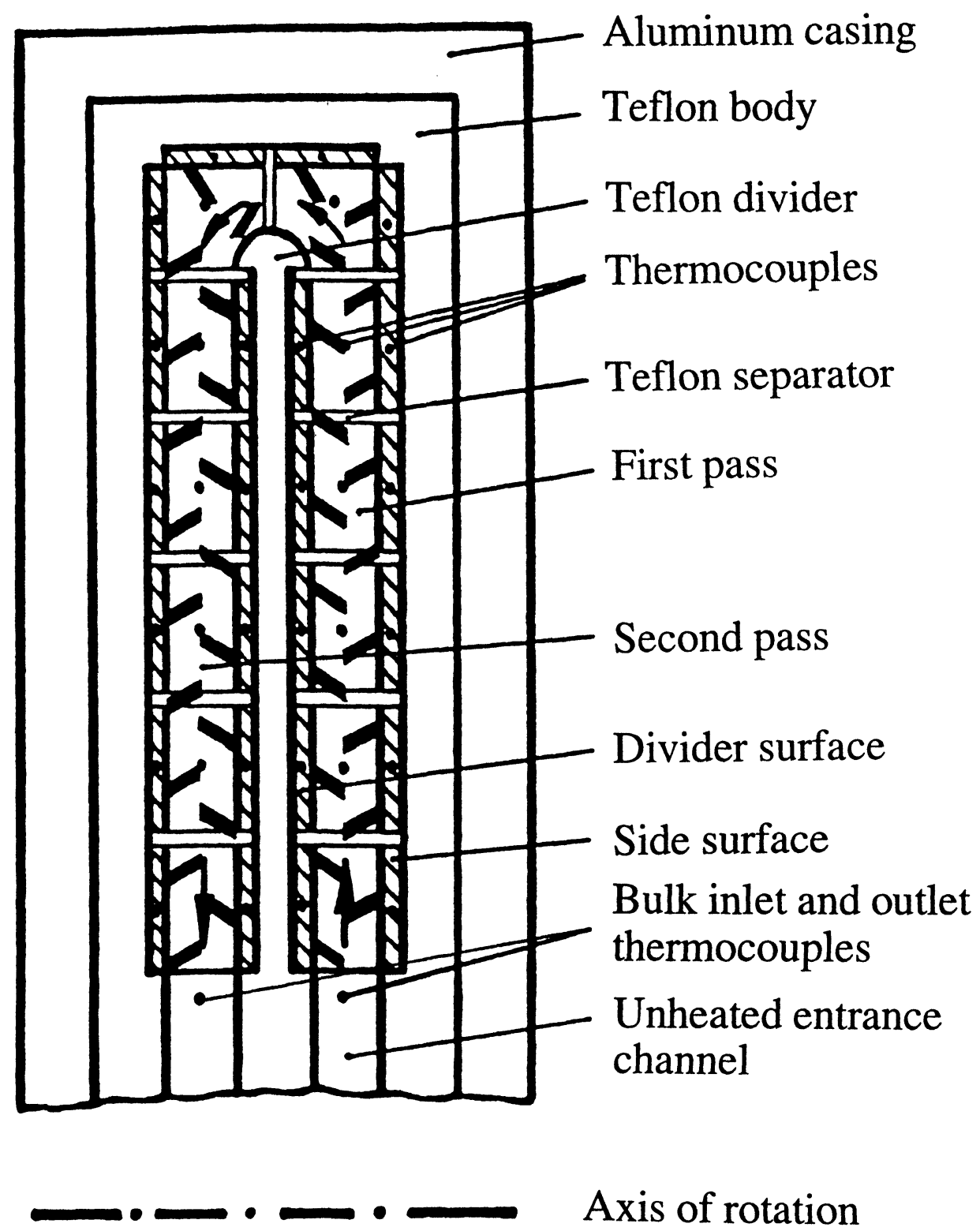

FIGURE 5 Cut-away view of the test section with $60^{\circ}$ staggered ribs. 

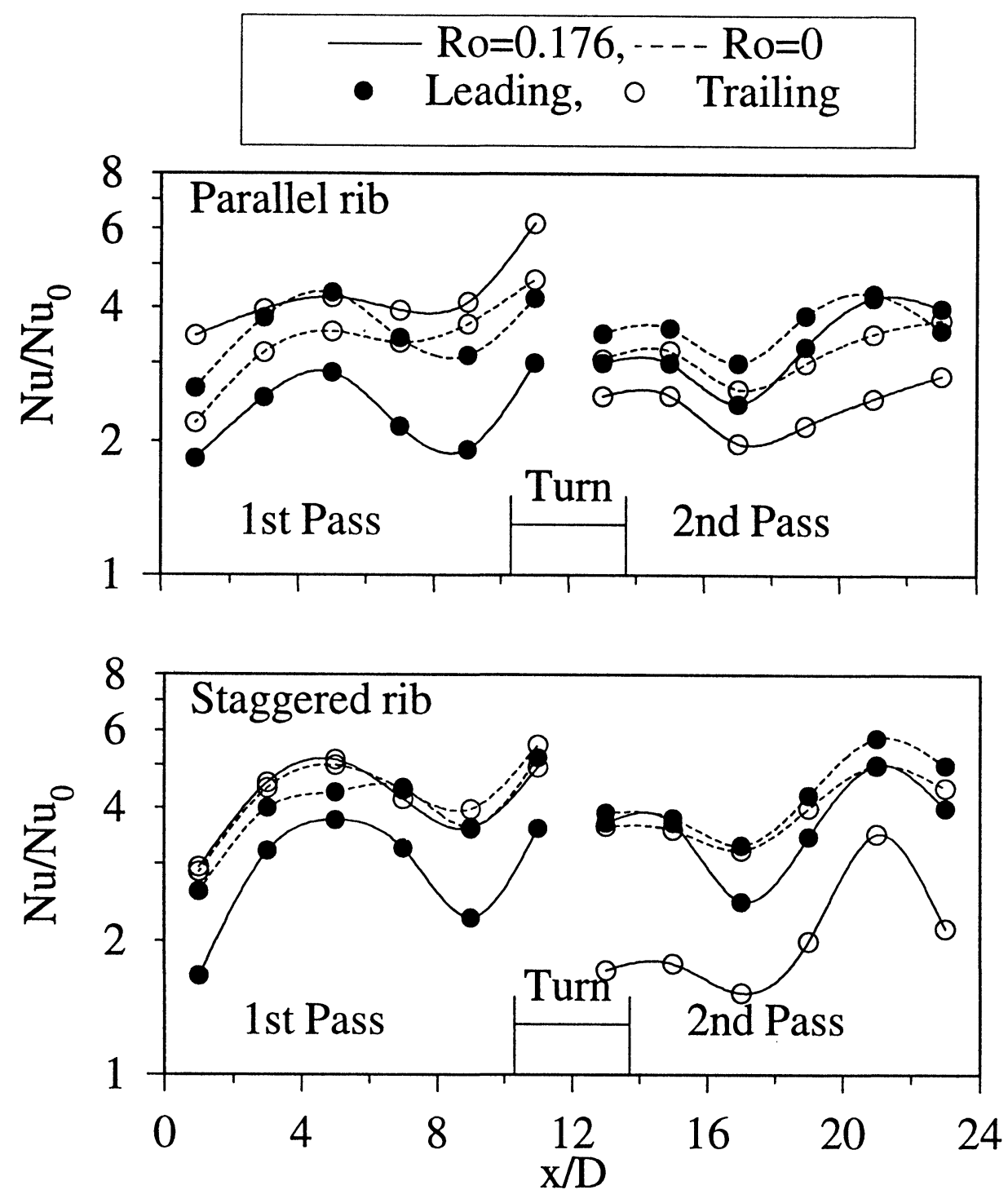

FIGURE 6 Local Nusselt number distribution for $\operatorname{Re}=\mathbf{5 , 0 0 0}$.

orientation may also be responsible for the initial drop in Nusselt number ratio. Similar to the first pass, the Nusselt number increases downstream of the inlet of the second pass as the secondary flow induced by the ribs starts to develop after the influences from the $180^{\circ}$ bend are dampened. Fully developed heat transfer results are not observed because of the short channel length and sharp turn in the flow passage.

Figure 6 shows that the difference of Nusselt number ratios between leading and trailing sides increase with rotation, although the distribution patterns remain similar to those about the stationary duct. The drop in firstpass leading and second-pass trailing Nusselt numbers are more than in the other two-ribbed surfaces. Johnson et al. [1994] observed similar behavior with $45^{\circ}$ skewed ribs. In an earlier study, Rohte and Johnston [1979] experimentally demonstrated that the turbulent shear layer past a backstep for low blockage ratio is more affected under stabilizing rotation (leading side of radially outward flow) than destabilizing rotation (trailing side of radially outward flow). The changes in the first-pass leading and trailing sides are similar to a smooth channel (Han et al. [1993]). The leading-side Nusselt number ratio decreases and trailing-side Nusselt number ratio increases with rotation. However, the second-pass results show a reduction in Nusselt number ratio in both sides with rotation. The 

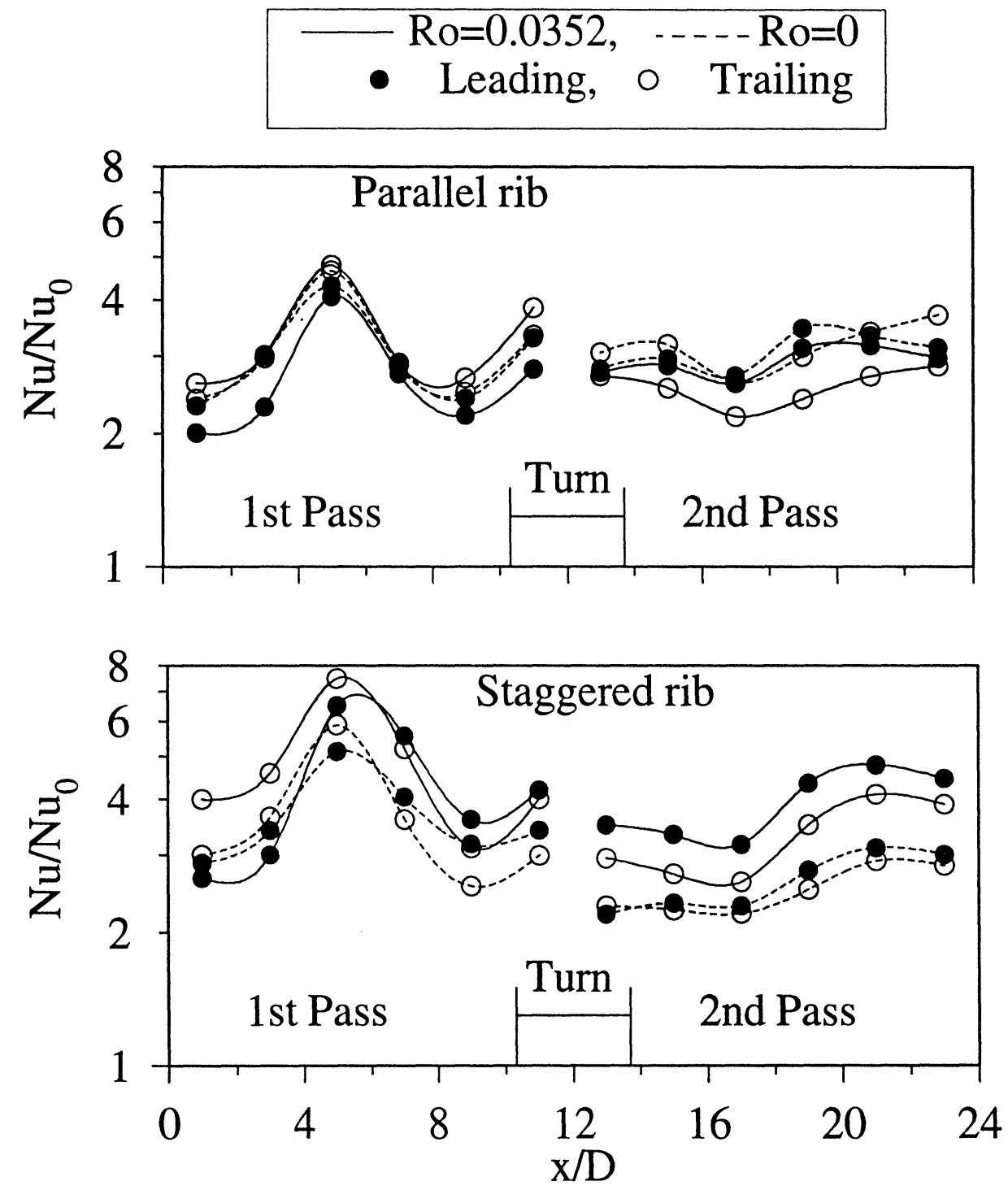

FIGURE 7 Local Nusselt number distribution for $\operatorname{Re}=25,000$.

second-pass leading side Nusselt number ratios are higher than the second-pass trailing side Nusselt number ratios. The Coriolis force in rotation sets up secondary flow in the rotating duct that causes an increase in Nusselt number for the first-pass trailing and the second-pass leading surfaces, and a decrease in Nusselt number for the first-pass leading and second-pass trailing surfaces.

Beside the Coriolis force in heated rotating ducts, the centrifugal force induces buoyancy effects that can play a major role in the heat transfer patterns. In the first pass, the velocity and turbulence at the inlet of the test section are higher near the trailing side than the leading side due to the Coriolis effect in the unheated starting length. Air at higher velocity regions remains cooler and denser than the lower velocity region. Therefore, air near the trailing side gets accelerated by a higher centrifugal buoyancy force and air near the leading side decelerates to maintain the same mass flow rate through the duct. Therefore, both the rotational buoyancy force and Coriolis force in the first pass increase the heat transfer from the trailing side and decrease heat transfer from the leading side. Unlike the first pass, the second pass has radially inward flow. The centrifugal buoyancy in the second pass accelerates the warmer low-velocity air and decelerates the denser highvelocity air. It can be observed from the Nusselt number profiles of Figure 6 , that the effects of rib orientations and 
Coriolis force in the second pass are clearly present. This is because the Nusselt number distribution profiles with rotation are similar to the nonrotating condition and the trailing-side Nusselt numbers are lower than the leadingside Nusselt numbers. However, the decrease in Nusselt number with rotation for the ribbed sides must be significantly influenced by the centrifugal buoyancy force. It can be argued that the rotational buoyancy accelerates the warmer fluid near the two smooth walls and, as a result, there is a reduction in velocity and heat transfer near and from the ribbed sides.

Figure 7 shows the results of $\operatorname{Re}=25,000$. The Nusselt number ratio distributions are similar to those for $\operatorname{Re}=5,000$ (Figure 6). The main difference observed with rotation is a significant increase in Nusselt number ratio in the second pass and in most of the first pass for staggered ribs. The effect of rotation for $\mathrm{Re}=25,000$ where the rotation number is less ( $\mathrm{Ro}=0.0352$ ) compared to $\operatorname{Re}=5,000(\operatorname{Ro}=0.176)$ is expected to be smaller. A possible reason for the enhancement of Nusselt number with rotation for staggered ribs can be a result of the interaction among the different secondary flow structures. Dutta et al. [1994] numerically showed that the vortex structure formed by rotation in a square channel at different rotational situations, can vary between four and two vortex structures. Figure 3 showed the schematic vortex structures generated by rib configurations and rotation. It is possible that the rotation induces a four-vortex structure for staggered ribs at Ro $=0.0352$. The four-vortex structure of the staggered rib (no-rotation) increases the core flow in the channel. The four-vortex structure of rotation opposes and reduces the vortex effect of the staggered rib. Therefore, it can be argued that as more air from the core gets redistributed toward the walls of the inter-rib region, the Nusselt number increases with rotation from a no-rotation situation.

Figure 8 shows the differences in Nusselt number ratios between the trailing and the leading sides. Smooth duct data (Han et al. [1993]) are included in this figure for comparison. The ribbed ducts show larger differences than smooth duct at high rotation number $(\mathrm{Ro}=0.352)$. It should be noted that although the differences are larger in ribbed ducts, the ratios of the Nusselt numbers for the two sides $\left(\mathrm{Nu}_{\text {trailing }} / \mathrm{Nu}_{\text {leading }}\right)$ are less for ribbed ducts than the smooth rotating channel. The difference between trailing- and leading-side Nusselt number ratios gradually approaches zero with a reduction in rotation number. The smooth duct data show the effects of vortical structure and difference of turbulence levels in leading and trailing sides from the rotational effects (Johnston et al. [1972]). Ribbed ducts also include the effects on separation process from higher and lower turbulence levels at pressure and suc- tion sides, respectively. The influence of secondary flows generated by skewed rib configurations on flow separation are more complicated. Centrifugal buoyancy force in the first pass (radially outward flow), tries to widen the differences between trailing- and leading-side heat transfer rates. Centrifugal buoyancy in the second pass (radially inward flow) tries to equate the heat transfer from two sides. Figure 8 shows that the first-pass differences for smooth duct are always higher than second-pass differences. The other two rib configurations follow this trend.

Figures 9 and 10 show the effect of rotation number on heat transfer. Figure 9 also shows local effects under rotation, which include Coriolis effect, centrifugal buoyancy effect, effects from secondary flows generated from rib orientations, and the effect of $180^{\circ}$ bend on Nusselt number ratio. Rather than show a uniform increase or decrease with rotation number, as observed in smooth rotating channels (Han et al. [1993]), there are variations of the Nusselt number ratios with the rotation numbers. Sides with stabilizing rotation (first-leading and second-trailing) generally show a reduction in Nusselt number ratio with an increase in rotation number. The other two ribbed sides show an opposite trend.

In Figure 10 the surface averaged Nusselt number ratios are plotted for four-rib bearing sides of the two-pass channel. Results from the nonrotating $\operatorname{Re}=25,000$ are plotted for the stationary case $($ Ro $=0)$. With an increase in rotation number the first-pass leading and second-pass trailing show mostly a decrease in Nusselt number ratio, whereas, the first-pass trailing and second-pass leading show mostly an increase in average Nusselt number ratio with rotation number. Earlier-published results of $90^{\circ}$ transverse (Parsons et al. [1994]) and $60^{\circ}$ parallel ribs (Zhang et al. [1993b], $60^{\circ}$ parallel ribs oriented differently in two passes) are included for comparison. The present $60^{\circ}$ parallel rib does better in the suction sides of the two passes (first-leading and second-trailing) than the previous $60^{\circ}$ parallel rib configuration, which shows higher Nusselt number ratios on the other two ribbed sides. The staggered rib shows a sharp increase in average Nusselt number ratios at low rotation number from the stationary duct results. As discussed earlier, this can be from a favorable secondary flow vortex structure induced by rotation. For $90^{\circ}$ transverse and $60^{\circ}$ parallel ribs the rate of increase of the average Nuselt number ratios in the second-pass leading is less than for the first-pass trailing. Whereas, for $60^{\circ}$ staggered ribs the rate of increase of the average Nusselt number ratios with rotation is similar for the first-pass trailing and the second-pass leading surfaces. Compared to the skewed ribs, the performance of the $90^{\circ}$ rib is worse on the first-pass leading and the second-pass trailing. The first-pass leading and the second-pass trail- 

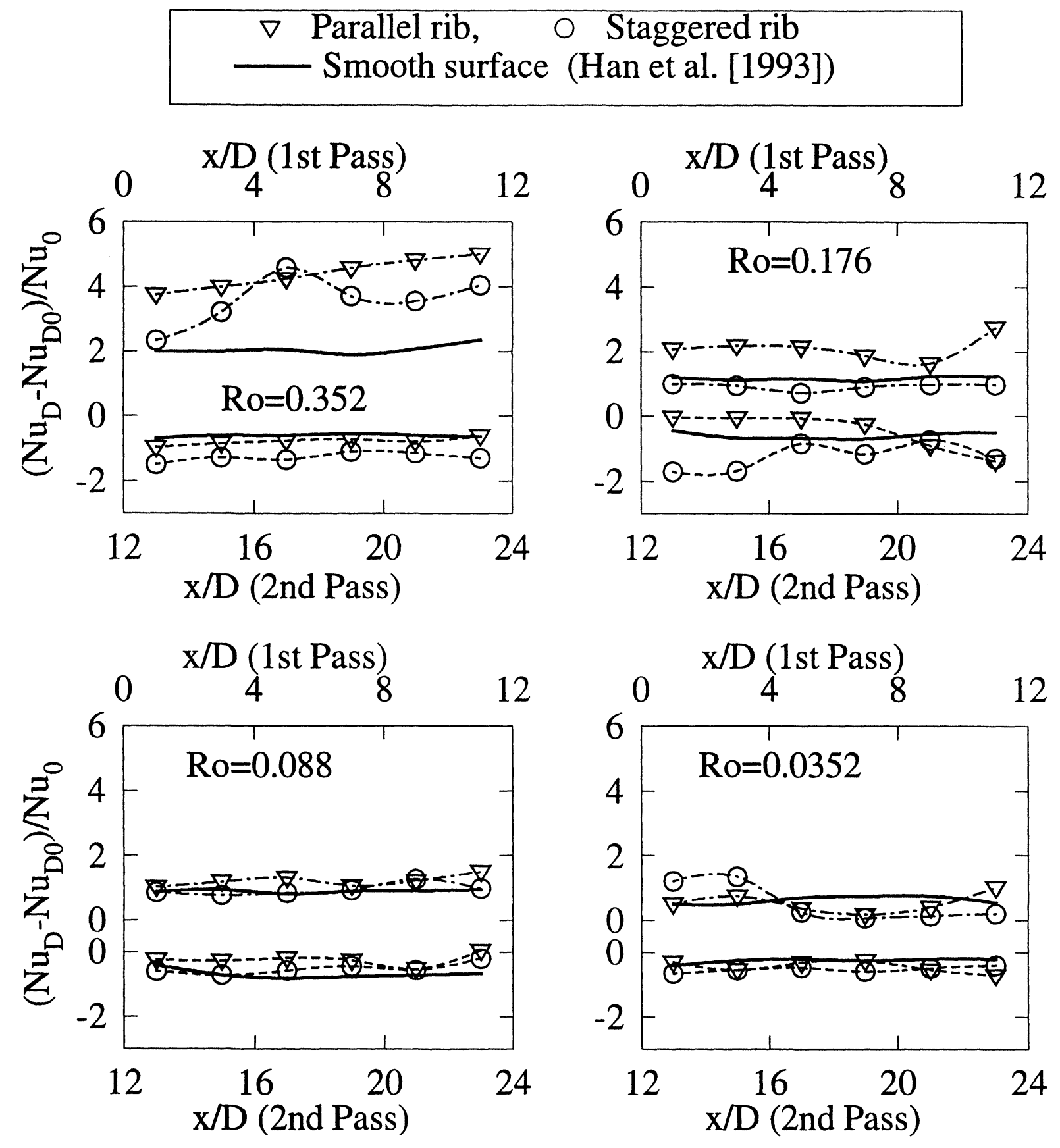

FIGURE 8 Differences between local Nusselt number ratios at different rotation numbers.

ing are the more important sides due to their lower heat transfer coefficients.

Figure 9 shows that the trailing-side Nusselt number ratio in the first pass $(x / D=5$ and 9$)$ increases with Ro and the leading-side Nusselt number ratio decreases with Ro at higher rotation numbers. Though the trailing-side Nusselt number ratio at $x / D=9$ for the parallel rib is higher than the staggered rib at a higher rotation number, the leading-side Nusselt number ratios for the staggered rib are higher than the parallel rib results. The surface averaged Nusselt number of Figure 10 showed the leading side of the first pass has higher Nusselt number ratios with staggered ribs. The differences between the leading- and trailing-side Nusselt numbers in Figure 8 are less for the 


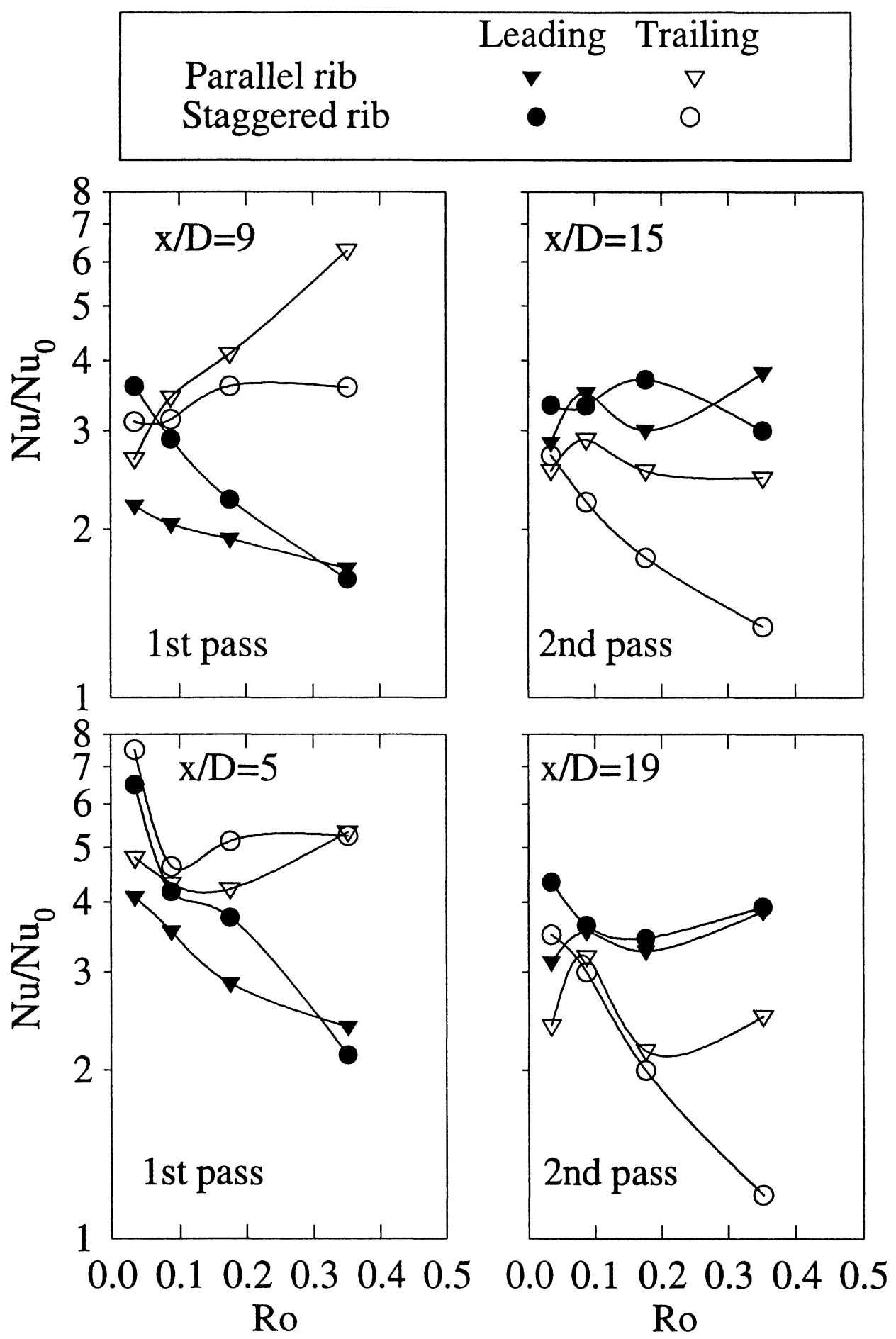

FIGURE 9 Effect of rotation number on Nusselt number ratios for the two rib configurations at selected axial locations. 


\section{$\nabla$ Parallel rib, $\bigcirc$ Staggered rib, - $90^{\circ}$ rib (Parsons et al. [1994]), Parallel rib (Zhang et al. [1993b])}
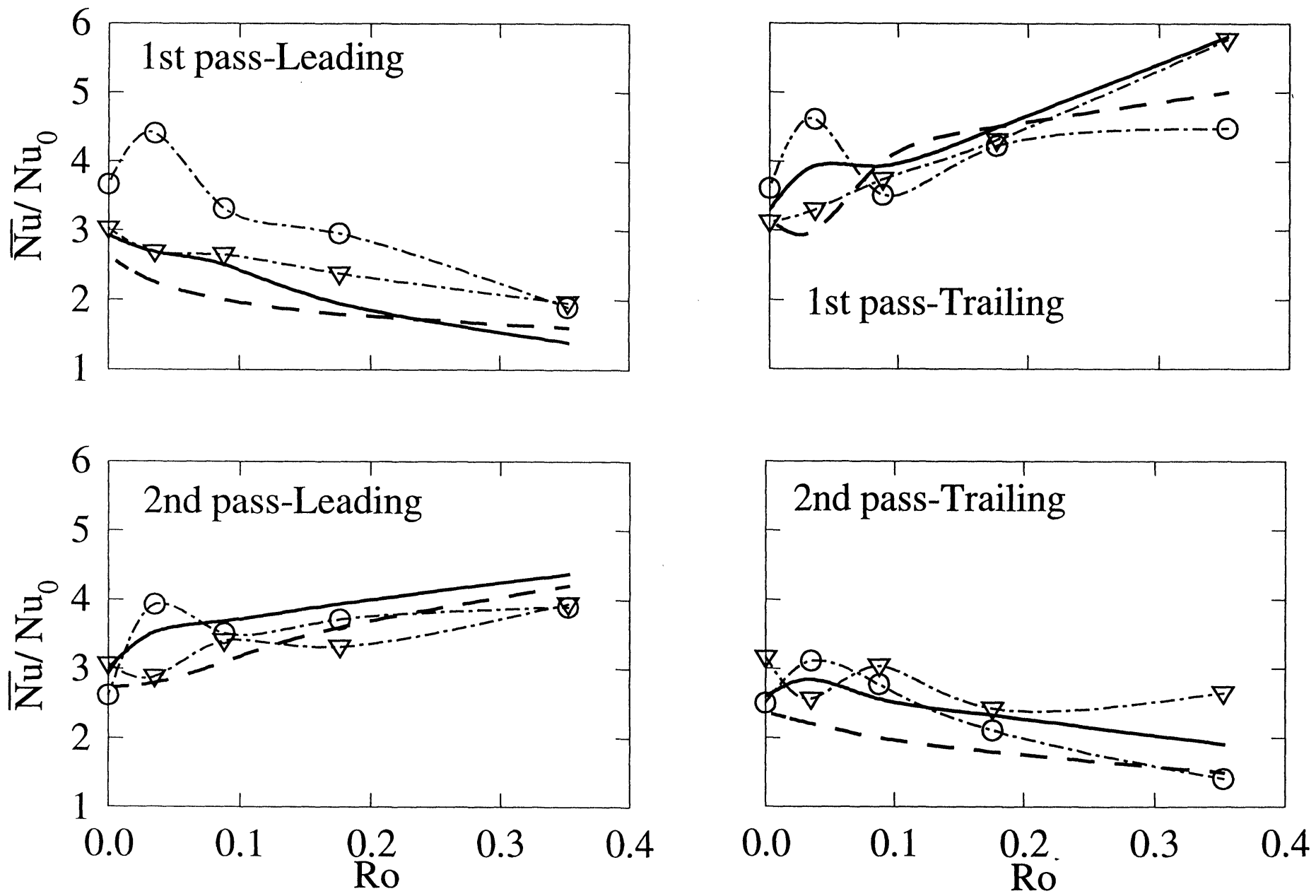

FIGURE 10 Surface averaged Nusselt number ratios for ribbed surfaces.

staggered rib in most situations in the first pass than the $60^{\circ}$ parallel rib. Therefore, a staggered rib is more suitable in the first pass than a $60^{\circ}$ parallel rib.

The second-pass $(x / D=15$ and 19) Nusselt number ratios of Figure 9 showed that $60^{\circ}$ parallel ribs are more advantageous than the staggered rib. The leading-side results for the staggered rib are better than the parallel rib, but the trailing-side Nusselt number ratios for the parallel rib are higher than the staggered rib results at higher rotation numbers. The surface averaged Nusselt number ratios of Figure 10 favors' the parallel rib. The surface averaged Nusselt number ratios for the second-pass trailing are higher for the parallel rib than the staggered rib. The differences in Nusselt numbers between the leading and the trailing surfaces for the parallel rib is less than the smooth surface (Figure 8). Therefore, the $60^{\circ}$ parallel rib is more suitable in the second pass than the staggered rib.

\section{CONCLUSION}

The present work focuses on an experimental study of heat transfer from rotating ribbed surfaces. Two types of 
ribs $\left(60^{\circ}\right.$ parallel and $60^{\circ}$ staggered $)$ are used, and the results are compared with previous results of $90^{\circ}$ transverse ribs, $60^{\circ}$ parallel ribs, and smooth wall results. The experimental work is carried out on a two-pass square duct that has both radially outward and inward flow situations. It is concluded that, the better heat transfer results with $60^{\circ}$ skewed ribs over standard $90^{\circ}$ transverse ribs in a rotating duct at moderate-to-high rotation numbers are as significant as a stationary duct at the first-pass leading and the second-pass trailing surfaces, which are the most important surfaces for heat transfer. Comparative studies of the two rib configurations $\left(60^{\circ}\right.$ parallel and $60^{\circ}$ staggered) suggest that the staggered rib is more suitable to augment heat transfer with rotation for the first pass and the $60^{\circ}$ parallel rib for the second pass.

\section{Acknowledgments}

This investigation was supported by the Texas Higher Education Coordinating Board (Energy Research in Application Program, 999903-050).

\section{Nomenclature}

D hydraulic diameter, $0.0127 \mathrm{~m}$

$h \quad$ heat transfer coefficient, $w / \mathrm{m}^{2}{ }^{\circ} \mathrm{C}$

$\mathrm{Nu} \quad$ Nusselt number, $h D / k$

$\overline{\mathrm{Nu}} \quad$ surface averaged Nusselt number

$\mathrm{Nu}_{D} \quad\left(\mathrm{Nu}_{\text {Trailing }}-\mathrm{Nu}_{\text {Leading }}\right)$ with rotation

$\mathrm{Nu}_{\text {Do }} \quad\left(\mathrm{Nu}_{\text {Trailing }}-\mathrm{Nu} \mathrm{u}_{\text {Leading }}\right)$ no rotation

$\mathrm{Nu}_{o} \quad$ Nusselt number in fully developed turbulent pipe flow

$q_{\text {net }} \quad$ net heat flux, w/ $\mathrm{m}^{2}$

$\operatorname{Re} \quad$ Reynolds number, $V D / v$

Ro rotation number, $\Omega D / V$

$T_{b} \quad$ bulk temperature, ${ }^{\circ} \mathrm{C}$

$T_{w} \quad$ local wall temperature, ${ }^{\circ} \mathrm{C}$

$V$ average flow velocity, $\mathrm{m} / \mathrm{s}$

$x \quad$ distance measured along the duct axis from the start of heating, $m$

\section{Greek Letters}

$v \quad$ kinematic viscosity of air, $\mathrm{m}^{2} / \mathrm{s}$

$\Omega$ rotational speed of the two-pass channel, $\mathrm{rad} / \mathrm{s}(1 \mathrm{rpm}=0.105$ $\mathrm{rad} / \mathrm{s})$

\section{References}

Clifford, R. J., Morris, W. D., and Harasgama, S. P., 1984. An Experimental Study of Local and Mean Heat Transfer in a Triangular-Sectioned Duct Rotating in the Orthogonal Mode, ASME Transactions, Journal of Engineering for Gas Turbines and Power, Vol. 106, pp. 661-667.

Dutta, S., Andrews, M. J., and Han, J. C., 1994. Numerical Prediction of Turbulent Heat Transfer in a Rotating Square Duct with Variable Rotational Buoyancy Effects, ASME HTD_Vol. 271, General Papers in Heat and Mass Transfer, Insulation, and Turbomachinery, edited by S. H. Chan et al., pp. 161-170.
Guidez, J., 1989. Study of the Convective Heat Transfer in Rotating Coolant Channels, ASME Journal of Turbomachinery, Vol. 111, pp. 43-50.

Han, J. C., 1984. Heat Transfer and Friction in Channels with Two Opposite Rib-Roughened Walls, ASME Journal of Heat Transfer, Vol. 106, pp. 774-781.

Han, J. C., Park, J. S., and Lei, C. K., 1985. Heat Transfer Enhancement in Channels with Rib Turbulence Promoter, ASME Journal of Engineering for Gas Turbines and Power, Vol. 107, pp. 629-635.

Han, J. C., Zhang, Y. M., and Lee, C. P., 1991. Augmented Heat Transfer in Square Channels with Parallel, Crossed, and V-Shaped Angled Ribs, Journal of Heat Transfer, Vol. 113, pp. 590-596.

Han, J. C., and Zhang, Y. M., 1992. Effect of Uneven Wall Temperature on Local Heat Transfer in a Rotating Square Channel With Smooth Walls and Radial Outward Flow, Journal of Heat Transfer, Vol. 114, No. 4, pp. 850-858.

Han, J. C., Zhang, Y. M., and Kalkuehler, K., 1993. Uneven Wall Temperature Effect on Local Heat Transfer in a Rotating Two-Pass Square Channel with Smooth Walls, Journal of Heat Transfer, Vol. 115, No. 4, pp. 912-920.

Han, J. C., Zhang, Y. M., and Lee, C. P., 1994. Influence of Surface Heating Condition on Local Heat Transfer in a Rotating Square Channel with Smooth Walls and Radial Outward Flow, ASME Journal of Turbomachinery, Vol. 116, No. 1, pp. 149-158.

Harasgama, S. P., and Morris, W. D., 1988. The Influence of Rotation on the Heat Transfer Characteristics of Circular, Triangular, and Squaresectioned Coolant Passages of Gas Turbine Rotor Blades, ASME Journal of Turbomachinery, Vol. 110, pp. 44-50.

Johnson, B. V., Wagner, J. H., Steuber, G. D., and Yeh, F. C., 1994. Heat Transfer in Rotating Serpentine Passages with Trips Skewed to the Flow, ASME Journal of Turbomachinery, Vol. 116, No. 1, pp. 113123.

Johnston, J. P., Halleen, R. M., and Lezius, D. K., 1972. Effect of Spanwise Rotation on the Structure of Two-Dimensional Fully Developed Turbulent Channel Flow, Journal of Fluid Mechanics, Vol. 56, part 3, pp. 533-557.

Kline, S. J., and McClintock, F. A., 1953. Describing Uncertainties in Single-Sample Experiments, Mechanical Engineering, pp. 3-8.

Mori, Y., Fukada, T., and Nakayama, W., 1971. Convective Heat Transfer in a Rotating Radial Circular Pipe (2nd Report), International Journal of Heat and Mass Transfer, Vol. 14, pp. 1807-1824.

Morris, W. D., and Ghavami-Nasr, G., 1991. Heat Transfer Measurements in Rectangular Channels with Orthogonal Mode Rotation, ASME Journal of Turbomachinery, Vol. 113, pp. 339-345.

Parsons, J. A., Han, J. C., and Zhang, Y. M., 1994. Wall Heating Effect on Local Heat Transfer in a Rotating Two-Pass Square Channel with $90^{\circ} \mathrm{Rib}$ Turbulators, International Journal of Heat and Mas Transfer, Vol. 37, No. 9, pp. 1411-1420.

Rohsenow, W. M., and Choi, H., 1961. Heat, Mass and Momentum Transfer, pp. 192-193, Prentice-Hall, Englewood Cliffs, NJ.

Rohte, P. H., and Johnston, J. P., 1979. Free Shear Layer Behavior in Rotating Systems, Journal of Fluids Engineering, Vol. 101, pp. 117120.

Soong, C. Y., Lin, S. T., and Hwang, G. J., 1991. An Experimental Study of Convective Heat Transfer in Radially Rotating Rectangular Ducts, Journal of Heat Transfer, Vol. 113, pp. 604-611.

Taslim, M. E., Rahman, A., and Spring, S. D., 1991a. An Experimental Investigation of Heat Transfer Coefficients in a Spanwise Rotating Channel with Two Opposite Rib-Roughened Walls, ASME Journal of Turbomachinery, Vol. 113, pp. 74-82.

Taslim, M. E., Bondi, L. A., and Kercher, D. M., 1991b. An Experimental Investigation of Heat Transfer in an Orthogonally Rotating Channel 
Roughened with 45 Degree Criss-Cross Ribs on Two Opposite Walls, ASME Journal of Turbomachinery, Vol. 113, pp. 346-353.

Wagner, J. H., Johnson, B. V., and Hajek, T. J., 1991a. Heat Transfer in Rotating Passages with Smooth Walls and Radial Outward Flow, ASME Journal of Turbomachinery, Vol. 113, pp. 42-51.

Wagner, J. H., Johnson, B. V., and Kopper, F. C., 1991b. Heat Transfer in Rotating Serpentine Passages with Smooth Walls, ASME Journal of Turbomachinery, Vol. 113, pp. 321-330.

Wagner, J. H., Johnson, B. V., Graziani, R. A., and Yeh, F. C., 1992. Heat Transfer in Rotating Serpentine Passages with Trips Normal to the Flow, ASME Journal of Turbomachinery, Vol. 114, pp. 847-857.
Yang, W. J., Zhang, N., and Chiou, J., 1992. Local Heat Transfer in a Rotating Serpentine Flow Passage, Journal of Heat Transfer, Vol. 114, pp. 354-361.

Zhang, N., Chiou, J., Fann, S., and Yang, W. J., 1993a. Local Heat Transfer Distribution in a Rotating Serpentine Rib-Roughened Flow Passage, Journal of Heat Transfer, Vol. 115, pp. 560-567.

Zhang, Y. M., Han, J. C., Parsons, J. A., and Lee, C. P., 1993b. Surface Heating Effect on Local Heat Transfer in a Rotating Two-Pass Square Channel with $60^{\circ}$ Angled Rib Turbulators, ASME 93-GT-336, accepted for publication in the ASME Journal of Turbomachinery. (1995). 

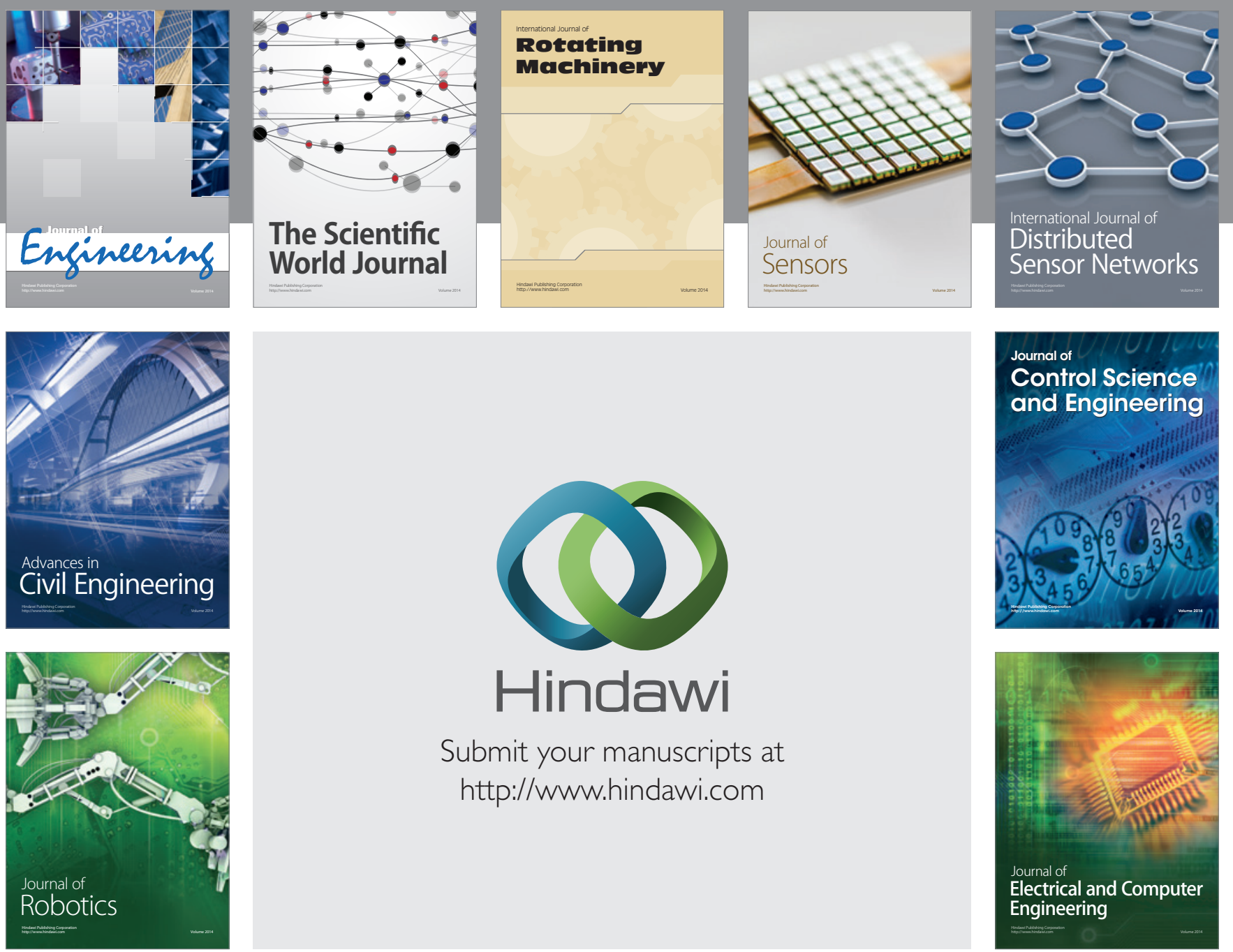

Submit your manuscripts at

http://www.hindawi.com
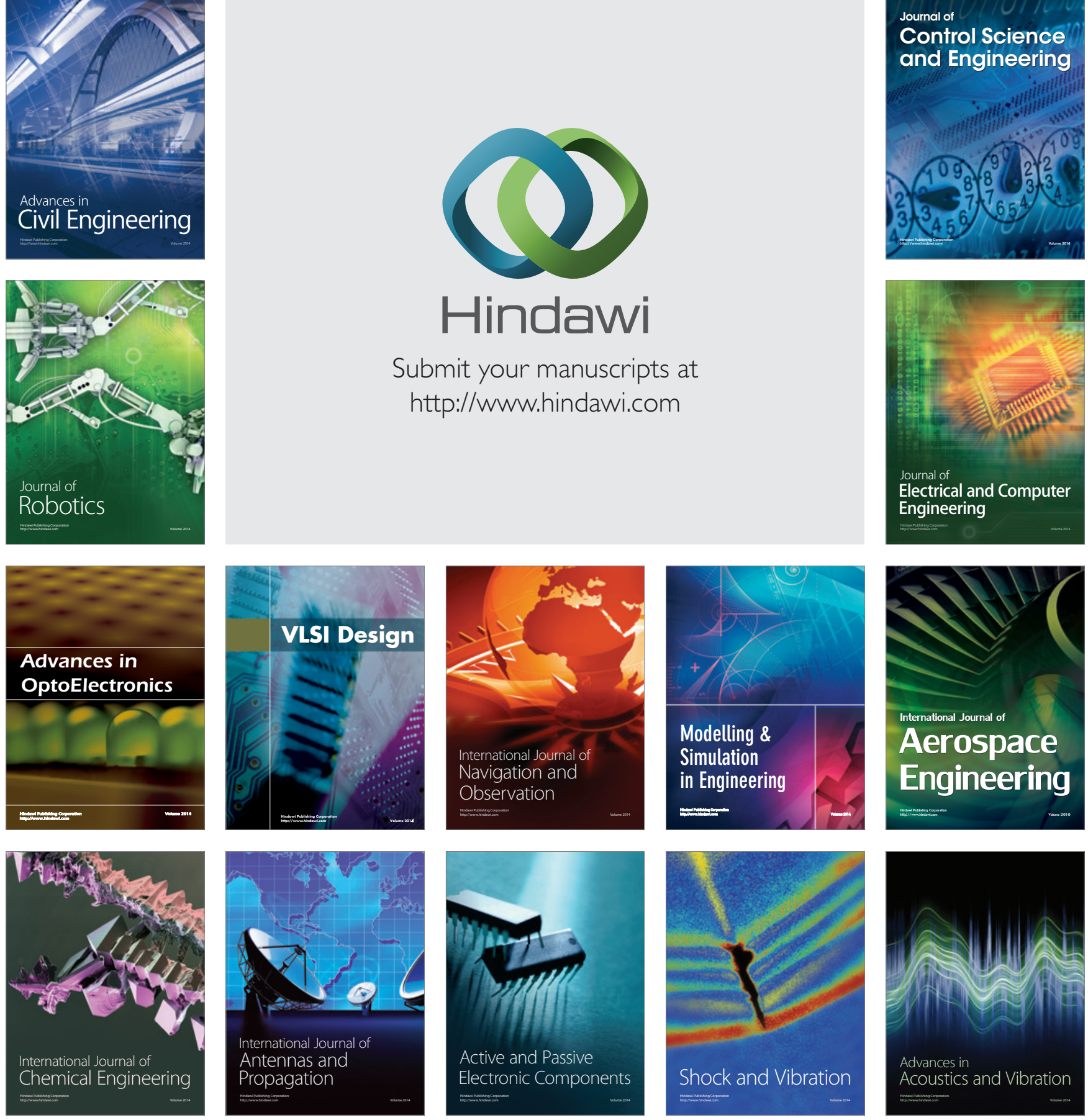